\title{
Infección por Helicobacter pylori y daño gástrico en niños de nivel socioeconómico bajo
}

\author{
Fernando Sarmiento Q. '; Eduardo Chávez C. ${ }^{2}$; Bárbara Pizarro M. ${ }^{2}$; \\ Elena Kakarieka W.; María Teresa Vial P..; Martín Gotteland R. ${ }^{3}$
}

\begin{abstract}
Resumen
Obpetivo: eslablecer relociones entre los hallazgos hislológicos y endoscópicos en la mucosa gástrica y la identificación de $H$. pylori en niños en que se realizó endoscopía digestiva por distinlas razones. Pacientes y método: Se estudiaron retrospectivamenle los anlecedentes clinicos, el aspecto endoscópico de la mucoso gástrico y los informes del examen histológico de la misma en 100 niños (59 varones) de baja condición sociócconómica, de 2 a 17 [promedio 11 ] años de edad en que, por diferentes motivos, fue necesorio reolizar endoscopía digestiva alta para Fines diognósticos y se colejaron con la presencia o ausencia de $H$. oylori registrada mediante prueba de la treasa o en el examen hislológico de las muestras obtenidas. Resultodos: En $09 \%$ de los niños de la serie se identificó $H$. pylori, cuyo prevalencia aumentó con la edad $\left(x^{2} 11,97 p=0,0025\right)$, llegando a $78 \%$ entre los 13 a 17 años. El agente se delectó en $30 \%$ de los niños sin lesiones histológicas y en $88 \%$ de los que tenion signos de gastritis. En $10 \%$ de los niños infectrdos no se registraban sínlomos de la alección a pesar de eslar coniaminados y rener lesiones gástricas. Conciusión: estos resuliados sugieren una fuerte asociación causa a efecto $\left(\chi^{2}=34,5, p=0,00001\right)$ entre colonización de la mucosa gástrica con el agenle y la existeneia de signos endoscópicos e histológicos de gastritis.
\end{abstract}

[Palobrat elave: Helicobacter pylori, gastritis, niños, nivel socioeconómico, prevalencia.)

\section{Helicobacter pylori infection and gastric damage in children of low socioeconomical status}

In people of low socioeconomical and defective higienic conditions, colonization of gastric mucosa by Helicobacter pylori is more frequent, occurs earlier in life and tends to produce chronic infection in adults. Objective: to conselate isolation of H. pylori from gastric mucosa to endoscopic and histologyc lindings. Potients and methods: endoscopic and histologic findings were relrospectively reloted to the isolation of $H$. pylori by urease (ClOl test or Giemsa stoining of samples from ontral gastric mucasa in 100 children 159 malel aged 2 to 17 -mean 11 - vears from low income families submited for diagnostic upper gastrointestinal endoscopic examination becouse of diferent causes. Results: $H$. pylori was delecled in $69 \%$ of the children and ils prevalence increased with age $\left(x^{2}=11.97, p=0.0025\right)$. Sixieen per cent of them were asynptomatic in spite of microbial isolation and the presence of mucosal gostric lesions. $H$. pylori was present in $30 \%$ of the patients without hislological lesions and in $88 \%$ of those with gastritis. Conclusions: o strong association between infection by $H$. pylori and endoscopic or histologic evidence of gastritis $\left(\chi^{2}-34.5, p=\right.$ $0.00001)$ is suggested.

\{Koy words: Helicobocter pylori, gastritis.)

1. Departamento de Pediatría, Universidad Nacional de Colombia. Bogota.

2. Unidad de Gastroenterología Infantil. Hospital Clínico San Borja-Arriarán y Departamento de Pediatria Centro, Facultad de Medicina Uajuersidad de Chile.

3. Unidad de Patología. Hospital Clínico San Borja-Arriarán.

4. Unidad de Gastcoenterología, Instituto Tecnológico de los Alimentos (INTA). Universidad de Chile.

Proyecro financiado en parte por el Servicio de Cooperación Cientifica del Laboratorio Saval. El Dr. Fernando Sarmiento fue beneficiario de una beca de la Universidad de Chile. Los resultados de este estudio fueron presentados al XXXVII Congreso Chileno de Pediatría (Puerto Natales, noviembre de 1996) y en la XXXIV Reunión de la Sociedad Latinoamericana de Investigación Pediátrica (SLAIP), Iguazú, Argentina, noviembre de 1996). 
Helicobacter pylori es una bacteria gram negativa, microaerofílica, que coloniza en forma específica la superficie de la mucosa gástrica. En las poblaciones de condiciones socioeconómica baja y de higiene deficiente. la infección frecuentemente ocurre en los primeros años de vida y persiste hasta la edad adulta' en forma crónica, de modo que 80 a $100 \%$ de los sujetos son portadores ${ }^{2}$. En las personas con mejor estándar de vida la infección es más tardía y la prevalencia en los aduitos no sobrepasa $70 \%{ }^{7}$. Chile ha sido descrito cono un país de transición epidemiológica ${ }^{4}$ pero la infección por $H$. pylori sigue siendo un importante problema de salud pública, pues su prevalencia se estima cercana a $40 \%$ en niños de 4 años y $70 \%$ en adolescentes ${ }^{5}$. La infección con este agente se considera la principal causa de gastritis y su presencia en el estómago se ha relacionado etiológicamente con la enfermedad ulcerosa, la gastritis atrófica y el aumento del riesgo de cáncer gástrico (ya sea linfomas o adenocarcinomas) ${ }^{6}$. Puesto que la incidencia de las afecciones malignas gástricas en Chile ${ }^{7}$ y en otros países del área ${ }^{8}$ es grande, el mejor conocimiento de la epidemiología y la fisiopatología de la infección puede contribuir significativamente al control de estas enfermedades. Nuestro propósito fue establecer relaciones entre la evidencia de colonización de la mucosa gástrica en niños y el aspecto endoscópico y microscópico de ella.

\section{Pacientes y Métodos}

Se analizaron retrospectivamente los infornes de las endoscopias gástricas realizadas a 100 niños (59 varones) de 2 a 17 (promedio II) años de edad, que consultacon entre agosto de 1995 y agosto de 1996 en la unidad de gastroenterologia pediátrica del Hospilal Clínico San Borja-Aıriarán, en Santiago metropolitano, por dolor abdominal recurrenke (DAR: $77 \%$ ), reflujo gastroesofígico o esolagilis (RGE/E. I0\%). hipertensión portal (HTP 3\%), sindrolne de malabsorción (SMA 3\%) y hemorragia digesliva alta (HDA 7\%). La endoscopía se realizó bajo sedación consciente con $0.2 \mathrm{mg} \cdot \mathbf{k g}$ de midazolan intravenoso (Do monid, Astra, Suecia) y 0 ,0l $\mathrm{ng} \cdot \mathrm{kg}$ de atropina. usando un endoscopio pediátrico Olympus XP-20 (Olympus Optical Co. Ltd., Tokio, Japón). Durante la endoscopia se tomaron dos biopsias del antro gástrico, La primera fue procesada para esludio anatomopalologico con tinción de hematoxilina-eosina y de Giemsa para la detección de $H$. prilori y la segunda se utiliźs para pesquisar su presencia mediante el prueba de ureasa (CLO). usando un estuche comercial (Laboratorio Linsan.
Santiago). Se consideró que el paciente estaba contaminado por el agente cuando el resultado de la histología o la prueba CLO eran positivos. En 84 pacientes se obtuvo también una biopsia duodenal. De acuerdo al sistema Sydney simplificado para adecuarlo a la realidad mís frecuentemente encontrada en pacientes pediátricos, los resultados endoscópicos fueron descritos como: gastritis eritematosa (hiperplasia nodular, eritema y exudado); gastritís erosiva; gastritis hemorrágica y úlcera. No se documentaron casos de gastritis por reflujo duodeno-gástrico, hiperplasia de pliegues y atrofia. Dentro del mismo sistema, la división histologica contempla gastritis aguda, gastritis crónica y formas especiales, con st correspondiente escala de severidad (ninguna, leve, moderada, severa). En la subdivisión morfológica: inflamación, actividad y Helicubacter pytori. Se excluyeron metaplasia intestinal y atrofia. Los resuttados se analizaron con las pruebas de $\chi^{2}$ y exacta de Fisher.

\section{Resultados}

H. pylori fue detectado en $69 \%$ de los niños estudiados, sin diferencias de sexo, pero con aumento significativo según edad (tabla 1). De $38 \%$ en los niños de 2.6 años, la tasa de colonización pasa a $75 \%$ en los niños de $7-12$ años de edad y a $78 \%$ en los de más de 13 años $\left(\chi^{2}\right.$ $11,97 p=0,0025)$. De los 16 niños asintomáticos (aquellos que no consultaron por DAR o por HDA), 11 estaban contaminados (6/11, 2/2 y $3 / 3$ de los niños con RGE/E, SMA e HTP, respectivamente).

Se observaron lesiones endoscópicas de la mucosa gástrica en 62 niños, en $46(74 \%)$ de los cuales se aisl 6 H. pylori (tabla 2). De los 38 niños con endoscopia normal, $23(61 \%)$ estaban. colonizados por el agente. Por lo tanto, la observación de lesiones endoscopicas no se asoció significativamente con la presencia de $H$. pylori $\left(\chi^{2}=2,06, p=0,15\right)$.

En 33 niños (tabla 2) la histología de la mucosa gástrica era normal y de ellos sólo $30 \%$ fueron $H$. pylori positivos. De los 67 niños con gastritis, $88 \%$ estaban colonizados. Esta diferencia es estadisticamente significativa $\left(\chi^{2}=34,5\right.$, $p=0,00001$ ).

De los 16 niños en quienes se realizó endoscopía digestiva alta por hipertensión portal, malabsorción y reflujo gastroesofágico o esofagitis, el examen histológico mostró signos de gastritis en 12 (75\%), de los cuales 11 tenían infección por $H$. pylori. Sólo el más joven de los pacientes menores de 6 años ( 2 años) estaba libre de daño histológico; otro niño de 3 años con 
Tabla 1

Frecuencia de colonización por

H. pylori por categorías de edad

\begin{tabular}{lccc}
\hline Edad & H.pylori (-) & H. pylori (+) & Total \\
\hline 56 años & $13(62 \%)$ & $8(38 \%)$ & 21 \\
7 a I 2 años & $11(25 \%)$ & $40(75 \%)$ & 51 \\
$\geq 13$ años & $7(22 \%)$ & $21(78 \%)$ & 28 \\
\hline Toral & 31 & 69 & 100 \\
\hline
\end{tabular}

$x^{2}=11.97, p=0.0025$

(-) aislamiento negativo. (+) aislamiento positivo

\section{Tabla 2}

Hallazgos endoscópicos e histológicos de la mucosa gástrica de niños contaminados (+) o no (-) por H. pylori

\begin{tabular}{llll}
\hline Endoscopía géstrica & $H . p y l o n(+)$ & $H . p y l o r i(-)$ & Total \\
\hline Normal & $23(6) \%)$ & $15(39 \%)$ & $38(100 \%)$ \\
Gastritis & $46(74 \%)$ & $16(26 \%)$ & $62(100 \%)$ \\
\hline
\end{tabular}

$\chi^{2}=2.06 p=0.15$

Histología gástrica

\begin{tabular}{lccc}
\hline $\begin{array}{l}\text { Normal } \\
\text { Gastritis, aguda }\end{array}$ & $10(30 \%)$ & $23(70 \%)$ & $33(100 \%)$ \\
y crónica & $59(88 \%)$ & $8(12 \%)$ & $67(100 \%)$
\end{tabular}

$x^{2}=\$ 4.5 \quad \mathrm{p}=0,0000 \mathrm{l}$

hemorragia digestiva alta tenía gastritis aguda y los demás gastritis crónica. Entre los 13 pacientes con resultados negativos para $H$. pylori de este grupo de edad, solo en uno se encontró daño de la mucosa en el examen histológico, en los dernás los exámenes endoscópicos $e$ histológicos eran normales.

El examen endoscópico de la mucosa duodenal era normal en 87 niños, de los cuales 60 $(69 \%)$ eran H. pytori positivos (tabla 3 ); 12 niños tenían duodenitis moderada a severa (de ellos 9 eran $H$. pylori positivos). En el duodeno no se encontró una asociación signifícativa entre la contaminación por $H$. pylori y la presencia de lesiones $\left(\chi^{2}=2,6, p=0,10\right)$ microscopicas.

\section{Comentario}

La participación del $H$, pylori en la patogenia de la enfermedad úlcero-péptica del adulto se ha reconocido en consensos internacionales ${ }^{10}$, sin embargo la que tiene en las afecciones gástricas pediátricas ha estado en constante discusión.

Estos resultados muestran una alta tasa de colonización por $H$. pylori en los niños estudiados, la que aumenta con la edad y se asocia fuertemente con gastritis crónica o aguda. A pesar del sesgo de selección por problemas gastroenterológicos, ellos coinciden con estudios de prevalencia obtenidas por serología que sugieren una de $40 \%$ en niños de 4 años y mayor a $60 \%$ en adolescentes ${ }^{\prime \prime}$.

La mayoría de los niños que participaron en este estudio provienen de familias de mala situación socioeconomica y deficientes condiciones higiénicas, las que se asocian con mayor contaminación microbiológica del am. biente, probablemente a través del ciclo fecaloral, y se traducen en colonización gástrica más precoz, intensa y persistente hasta la edad adulta ${ }^{12,13}$

La contaminación microbiologica ambiental también ha sido postulada como el origen de la enteropatía ambiental crónica ${ }^{\mathrm{I}}$, síndrome en e] cual se ha observado alteraciones morfofuncionales leves de la mucosa intestinal, atribuidas al

\section{Tabla 3}

Hallazgos endoscópicos e histológicos de la mucosa duodenal de niños contaminados (+) o no (-) por H. pylori

\begin{tabular}{lrrr}
$\begin{array}{l}\text { Endoscopí } \\
\text { duodeno }\end{array}$ & H.pylori (+) & H. pylori (-) & Total \\
\hline Noranal & $60(69 \%)$ & $27(31 \%)$ & $87(100 \%)$ \\
Duodenitis & $9(82 \%)$ & $2(18 \%)$ & $11(100 \%)$ \\
Duodenitis severa & 0 & $1(100 \%)$ & $1(100 \%)$ \\
Ulcera duodenal & 0 & $1(100 \%)$ & $1(100 \%)$ \\
\hline
\end{tabular}

$\chi^{2}=5.3 \mathrm{p}=0.15$

\section{Histología duodeno}

\begin{tabular}{llrl}
\hline Normal & $45(65 \%)$ & $24(35 \%)$ & $69(100 \%)$ \\
Duodenitis & $13(87 \%)$ & $2(13 \%)$ & $15(100 \%)$ \\
\hline
\end{tabular}

$x^{2}=2.6 p=0,1$ 
paso continuo pero asintomático de patógenos a través del tubo digestivo. En el caso de $H$. pylori la mayoría de los individuos contaminados por la bacteria permanecen asintomáticos a pesar de alteraciones leves de su mucosa gástrica, con bajo porcentaje de desarrollo de enferme$\mathrm{dad}^{15}$. Por estas razones se podría postular a la colonización por H. pylori como una gastropatía ambiental crónica, parecida a la descrita para el intestino delgado.

Nuestros resultados confirman la asociación significativa entre el daño histológico gástrico y la presencia de $H$. pylori; sin embargo ella es menos estrecha que en adultos ${ }^{16}$. La erradicación del microorganismo con antibióticos da como resultado la regresión de las lesiones de la mucosa ${ }^{17}$. En lesiones del duodeno la asociación es menor y no significativa como lo ilustran los casos de dos niños con duodenitis severa, úlcera duodenal y resultados negativos para H. pulori.

La ausencia de lesiones histológicas en niños en que se encontró infección por H. pylori, podría explicarse por colonización reciente y poco tiempo para que la bacteria provoque alteraciones, lo mismo que en el niño menor de 2 años. Otra explicación podría ser la contaminación con cepas de menor poder citotóxico (en términos de expresión de toxinas, por ejemplo) $)^{18}$.

La importante proporción de niños $(25 \%)$ en que no se identific $6 H$. pylori, pero tenían lesiones gástricas, podría estar relacionada con el método utilizado para detectar el agente, pues la prueba de ureasa y la tinción de Giemsa se efectúan en biopsias, con una cantidad mínima de tejido, y la colonización de la superficie de la mucosa por la bacteria no es homogénea sino circunscrita en áreas ${ }^{19}$, de modo que existe ba probabilidad de obtener muestras de sitios no colonizados. De hecho, no hay concordancia completa entre Ios resultados de la prueba CLO y la tinción histológica. Esta misma limitación podría explicar la pobre asociación entre daño histológico duodenal y presencia de $H$. pylori. También es posible que el paso continuo de patógenos a través del tubo digestivo en situaciones de alta contaminación ambiental, que altera la morfología de la mucosa intestinał, pueda dañar igualmente la mucosa gástrica en algunas personas.

\section{Referencias}

1. Klein PD. Gilman RH, Leon-Barua $R$, Diaz F, O'Brian Smirh E: The epidemiology of Helicobacter in Peruvian children between 6 and 30 months of age. Am J Gastroenterol 1994; 89: 2196-2200.

2. Malaty HM, Graham DY: Importance of childhood socioeconomic status on the current prevalence of Helicobacier pylori. Gut 1994: 35; 742-745.

3. Graham $D Y$, Malaty $H M$, Evans $E G$, Klein $P D$, Adam $E$ : Epidemiology of Helicobacter pylori in an asympcomatic population in the United States. Gastroenterology 1991: 100: 1495-150I.

4. Albala $C$, Vio $F$, Robledo A, Icaza $G$ : La transición epidemiologica en Chile. Rev Med Chile 1993; 121. 1446-1455.

5. Russell $R G$, Wasserman SS, O'Donnoghue SM: Serologic respond to Helicobacter pylori among children and teenagers in Northero Chile. Am J Trop Med Hyg 1993: 49: 189-191.

6. Forman $D$ : The prevalence of Helicobacter pylori in gastric cancer. Alim Phannacol Therap 1995: 9 (suppl 2): $71-76$.

7. Medina E: Las enfermedades digestivas en Chile. Panorama epidemiológico. Rev Med Chile 1988; 116 : 282-288.

8. Bernstein $M$. Monge $E$, León-Baría $R_{1}$ et al: Low peptic ulcer and high gastric cancer prevalence in a developing country with high prevalence of infection by Helicobacter pylori. J Clin Gastroenterol 1991; 13; 154-156.

9. Misiewice J. Tytgat GNJ. Goodwin CS, et al: The Sydney Sistem: A new classification of gastritis. Working Party Reports. World Congresses of Gastroenterology. Sydney Australia 1990: 1-10.

10. National Institute of Health, Helicobacter pylori in peptic ulcer disease. Consensus Development Conference Statement 1994: feb. 7-9; 12(1).

1l. Hopkins RJ, Vial PA. Ferreccio C. et al: Sero prevalence of Helicobacter pylori in Chile. Vegetables may served as one route of transmission. I Infect Dis 1993; 168: 222-226.

12. Sashar MA, Simjee AE. Wistenberg DF: Seroprevalence of Helicobacter pylori infection in NalKwaZulu South Aftica. Eur J Gastroenterol Hepatol 1994; 6: 37-41.

13. Sitas F, Forman D. Yarnel JWG: Helicobacter pylori infection rates in relation to age and social classs in a population of Welsh men. Gur 1991; 32: 25-28.

14. Brunser O. Araya M. Espinoza J: Chronic environmenial enteroparhy in a temperate climate. Clin Nutr 1987:41: 251-26]

15. Correa $P$ : The gastric microenvironment determines Helicobacter pylori colonization. Am J Gastroenterol 1995; 90: 1379-1381.

16. Hazell SL, Hennessy WB, Borady TJ, Carrick J. Raision M: Camplobacter pyloridis gastritis II: distribution of bacteria and asociated inflammation in the gastroduodenal envifonment. AIn I Gastroenterol 1987; 82: 297-301. 
17. Figterod G. Acuña $R$. Troncoso $M$, et al: Low $H$. pilori reinfection rate after triple therapy in chilean duodenal ulcer patients. Am J Gastroenterol 1996; 91 : 1395. 1399.

18. Crabtree JE, Farmery SM: Helicobacter pytori and gastric mucosal cytokines: evidence of a CagA. positive strains are more visulent. Lab Invest 1995: 73: 742-745.

19. Cutler AF. Havstad $S$, Chen $K$, Blaser $M J$, PérezPerez GI, Schubert T: Accuracy of invasive and non invasive tests to diagnose Helicobacter pylori infection. Gastroenterology 1995; 109: 136-14l.

Esta publicación está disponible en copias de microfilms de $16 \mathrm{y}$ $35 \mathrm{~mm}$ y microfichas de $105 \mathrm{~mm}$, las que pueden solicitarse a:

University Microfilms International 300 North Zeeb Road Ann Arbor, Michigan 48106, USA.

This journalis also available in $16 \mathrm{~mm}$ microfilm, $35 \mathrm{~mm}$ microfilm and $105 \mathrm{~mm}$ microfilm copies through

University Microfilms International, 300 North Zeeb Road, Ann Arbor, Michigan 48106, USA. 